\title{
Multiple Target Detection For OFDM Radar Based On Convolutional Neural Network
}

\author{
Jae-Woong Choi and Eui-Rim Jeong* \\ Graduate student, Department of Mobile Convergence and Engineering, Hanbat National University, Daejeon, \\ 34158, Republic of Korea. \\ *Professor, Department of Information and Communication Engineering, Hanbat National University, Daejeon, \\ 34158, Republic of Korea. \\ *Corresponding author. Email address: erjeong@ @anbat.ac.kr
}

\begin{abstract}
Article History:Received:11 november 2020; Accepted: 27 December 2020; Published online: 05 April 2021
Abstract: The objective of this paper is to propose a multiple target identification technique for orthogonal frequency division multiplexing (OFDM) radars. First, a 2-D (range \& Doppler) periodogram is obtained from the reflected signal through 2-D fast Fourier transform (FFT) of the received OFDM symbols. Usually, the peaks of the periodogram indicates the targets. Conventionally, peak search algorithms are used to find the multiple targets. In this paper, however, a convolutional neural network (CNN) classifier is proposed to identify the targets. The proposed technique does not need any additional information but the 2-D periodogram while the conventional method requires the noise variance as well as the periodogram. The performance is examined through computer simulation. According to the results, if the number of maximum identifiable targets are small, the proposed technique performs well. However, as the number increases, the detection accuracy decreases. In the simulation environments, the proposed method outperforms the conventional one. The proposed OFDM radar technique can be applied to $6 \mathrm{G}$ mobile communications to identify the moving targets around the transmitter without additional frequency resource for radar systems.
\end{abstract}

Keywords: CNN, OFDM radar, Target detection, Multiple targets, Direct coupling, Periodogram.

\section{Introduction}

Many technologies and applications demand higher communications capacity and bandwidth while the importance of various radio-based sensing schemes is also increasing in commercial, industrial and military fields [1]. The shortage of the available radio spectrum has started to raise interest in merging those communication and sensing functionalities to share the same frequency bands [2].

The integrated or joint operation of communications and radar systems implies that the same waveform is utilized for both tasks. It is well known that orthogonal frequency division multiplexing (OFDM) waveforms are well suited for radio communications, providing robustness against multipath fading and high flexibility in radio system design and resource management. The wideband OFDM waveform also satisfies the requirements as radar signals for fine resolution of range and Doppler estimation. Due to those reasons, the use of OFDM-based radar systems is receiving growing interest [1,2]. In [3], OFDM-based radar and communication system was developed and verified through experiments with commercial software defined radios (SDRs). In [4] and [5], the convergence of mobile communications and radar systems was addressed based on the use of the LTE and 5G NR waveform for radar purposes. The existing works only provide the feasibility of OFDM radar by simulations and experiments, but the detailed algorithm for finding the moving targets is not given. Recently, applying deep learning techniques in radar problem is gaining growing attention. The discrimination between vehicle, cyclist and pedestrian by using convolutional neural network $(\mathrm{CNN})$ was proposed for frequency modulated continuous wave (FMCW) radar [6], and similarly, a classification technique of real target, clutter, and dense multi-false targets is developed for radar systems based on factorized CNN in [7]. In [8,9], the CNN was used for enhancing the range-Doppler radar image. To the best of the authors' knowledge, multiple target detection problem for OFDM radar systems has not been dealt in the existing literatures.

In this paper, we propose multiple target identification technique for OFDM radar based on CNN. The procedure of the proposed technique is as follows. After cyclic prefix $(\mathrm{CP})$ removal and fast Fourier transform (FFT), the frequency domain received signal is obtained. Then, the signal is divided by the transmitted signal to remove modulation effect at the received signal. The same procedure is repeated on consecutive received OFDM symbols and the resulting signals are stacked to form a 2-D signal. Through 2-D FFT and taking magnitude square, the signal is converted into a 2-D range-Doppler periodogram. Usually, the local peaks of the periodogram indicates the targets, and the peaks' $\mathrm{x}$-axis and y-axis values indicate the distances and speeds of the targets, respectively. However, identifying the moving targets is challenging because of the direct coupling components between the transmitter and the receiver antennas. Conventional techniques find the local maxima first, and collect those bigger than a certain threshold. Then, the peaks close to zero Doppler frequency are

${ }^{*}$ Corresponding author: Eui-Rim Jeong*

Professor, Department of Information and Communication Engineering, Hanbat National University, Daejeon, 34158, Republic of Korea.

Email address: erjeong@ hanbat.ac.kr 
considered as direct coupling components and removed. The optimal threshold, however, requires the information on signal to noise ratio (SNR) and noise variance, which is difficult to obtain.

In the proposed technique, the number of targets is found via a CNN classifier, one of famous deep learning techniques [10]-[13]. Specifically, the 2-D periodogram is inputted to the proposed CNN and the output is the predicted number of targets. No information other than the periodogram is required. We propose two kinds of CNN inputs. The first one is one 2-D periodogram for the targets, and the second one is two 2-D periodograms. In the latter, one signal is a current 2-D periodogram for the target, and the other is for the direct coupling components only. This periodogram can be obtained only when there is no targets. For example, if the OFDM radar system is equipped in a base station, and it collects the periodogram at dawn, only direct coupling components can exist in the periodogram. The performance of the proposed technique is examined through computer simulation. According to the results, the proposed method is more than $4 \mathrm{~dB}$ superior to the conventional method in the SNR range of -20 to $10 \mathrm{~dB}$. If two periodograms are used for the CNN input, over 2 $\mathrm{dB}$ further improvement can be achieved.

\section{System and signal model}

OFDM radar system model considered in this paper is shown in Figure 1. The $m$-th transmitted signal vector is denoted by $\boldsymbol{c}_{m}=\left[c_{0, m}, c_{1, m}, \cdots c_{N-1, m}\right]^{T}$ where $N$ is FFT size. In general, $c_{n, m}$ is a complex symbol generated via channel coding and digital modulation of the transmitted information. However, those processing is not important for radar signal processing and omitted here for simplicity. The vector signal $\boldsymbol{c}_{m}$ is converted into a time domain signal through inverse FFT (IFFT) and transmitted after inserting a CP. The reflected signal from the targets is received and the CP is removed. Then, it is converted into frequency domain signal via FFT. The FFT output is denoted by $\boldsymbol{f}_{m}=\left[f_{0, m}, f_{1, m}, \cdots f_{N-1, m}\right]^{T} . \boldsymbol{c}_{m}$ and $\boldsymbol{f}_{m}$ are different due to the time delay and the Doppler frequency of the reflected signal. By comparing and processing $\boldsymbol{c}_{m}$ and $\boldsymbol{f}_{m}$, the targets are identified.

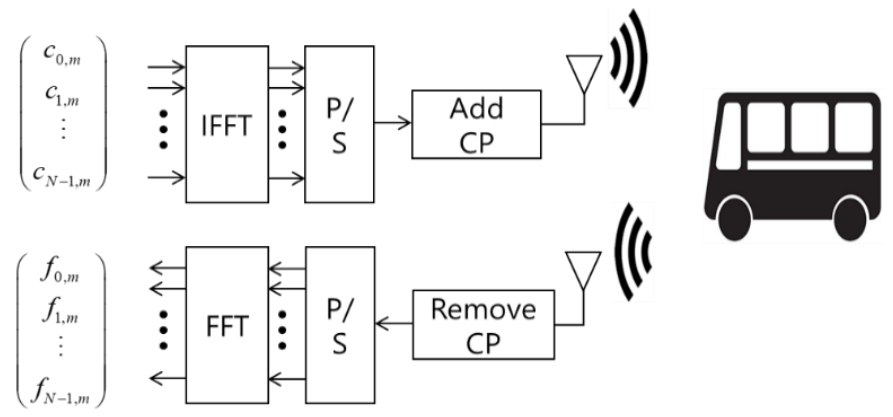

Figure 1 System model

If a totalMOFDM symbols are transmitted, the transmitted and the received signals are represented as (1) and (2), respectively.

$$
\begin{aligned}
\boldsymbol{C} & =\left[\begin{array}{cccc}
c_{0,0} & c_{0,1} & \cdots & c_{0, M-1} \\
c_{1,0} & c_{1,1} & \cdots & c_{1, M-1} \\
\vdots & \vdots & \ddots & \vdots \\
c_{N-1,0} & c_{N-1,1} & \cdots & c_{N-1, M-1}
\end{array}\right] \\
\boldsymbol{F}_{R} & =\left[\begin{array}{cccc}
f_{0,0} & f_{0,1} & \cdots & f_{0, M-1} \\
f_{1,0} & f_{1,1} & \cdots & f_{1, M-1} \\
\vdots & \vdots & \ddots & \vdots \\
f_{N-1,0} & f_{N-1,1} & \cdots & f_{N-1, M-1}
\end{array}\right]
\end{aligned}
$$

Every row of the matrix corresponds to the data on one subcarrier, whereas every column corresponds to the data on one OFDM symbol. It is assumed that the following parameters are known.

- The subcarrier space is $\Delta f$, and therefore the OFDM symbol duration is $T=1 / \Delta f$.

- The duration of the CP is $T_{G}$.

- The sampling rate after the IFFT is $f_{S}$ and $\Delta f=f_{S} / N$

- The carrier frequency is $f_{C}$

While transmitting, the receiver is active to pick up the reflected signal. It is important that the receiver is exactly synchronous to the transmitter, i.e., there must be no time or frequency offset. The received signal can be written as

$$
\begin{gathered}
\left(\boldsymbol{F}_{R}\right)_{k, l}=\sum_{h=0}^{H_{t}-1} b_{h}(\boldsymbol{C})_{k, l} e^{j 2 \pi T f_{D, h} l} e^{-j 2 \pi \tau_{h} \Delta f k} e^{j \phi_{h}} \\
+\sum_{i=0}^{H_{d}-1} b_{i}(\boldsymbol{C})_{k, l} e^{-j 2 \pi \tau_{i} \Delta f k} e^{j \phi_{i}}+(\widetilde{\boldsymbol{Z}})_{k, l}
\end{gathered}
$$


where $(\boldsymbol{A})_{k, l}$ represents the $(k, l)$-th element of the matrix $\boldsymbol{A}$, and $H_{t}$ denotes the number of reflecting targets. $(h+1)$-th target has a distance $d_{h}$ which translates into a delay $\tau_{h}\left(=d_{h} / c_{0}\right)$ where $c_{0}$ is the speed of light. $f_{D, h}$ is the Doppler frequency and $\phi_{h}$ is an unknown phase offset. The attenuation $b_{h}$ can be approximated by the point-scatter model [3].

$$
b_{h}=\sqrt{\frac{c_{0} \sigma_{R C S, h}}{(4 \pi)^{3} d_{h}^{4} f_{C}^{2}}}
$$

where $\sigma_{R C S}$ is the radar cross section. The second summation at the right hand side of (3) represent the direct coupling components, and the number of components is $H_{d}$. Direct coupling components are caused by direct coupling between Tx and Rx antennas or circuits. Therefore, the time delays or equivalent distances of the direct coupling components are close to zero. To simulate such direct coupling, the equivalent distances are generated following the Weibull probability density function (PDF):

$$
f\left(d_{i} ; \alpha, \beta\right)=\frac{\alpha}{\beta}\left(\frac{d_{i}}{\beta}\right)^{\alpha-1} e^{-\left(d_{i} / \beta\right)^{\alpha}}
$$

and the equivalent radar cross sections are generated uniformly random. The all direct coupling components have zero Doppler frequency. The matrix $\boldsymbol{Z} \in C^{N \times M}$ is white Gaussian noise. To remove the transmitted symbols in $\boldsymbol{F}_{R}$, element-wise division is performed to yield.

$$
\begin{gathered}
(\boldsymbol{F})_{k, l} \triangleq\left(\boldsymbol{F}_{R}\right)_{k, l} /(\boldsymbol{C})_{k, l} \\
=\sum_{h=0}^{H_{t}-1} b_{h} e^{j 2 \pi T_{O} f_{D, h} l} e^{-j 2 \pi \tau_{h} \Delta f k} e^{j \phi_{h}}+\sum_{i=0}^{H_{d}-1} b_{i} e^{-j 2 \pi \tau_{i} \Delta f k} e^{j \phi_{i}}+(\boldsymbol{Z})_{k, l}
\end{gathered}
$$

where $(\boldsymbol{Z})_{k, l}=(\widetilde{\boldsymbol{Z}})_{k, l} /(\boldsymbol{C})_{k, l}$. The radar problem is detection and identification of two sinusoid. The first exponential inside the summation in (5) contains the Doppler frequency and the second exponential has the time delay or distance. The radar cross section can be found from the magnitude. To separate and estimate the sinusoids for the targets, 2-D periodogram (magnitude square of discrete Fourier transform (DFT)) is used:

$$
(\boldsymbol{R})_{n, m}=\frac{1}{N M}\left|\sum_{k=0}^{N-1} \sum_{l=0}^{M-1}(\boldsymbol{F})_{k, l}(\boldsymbol{W})_{k . l} e^{-j 2 \pi\left(\frac{k n}{N_{F F T}}+\frac{l m}{M_{F F T}}\right)}\right|^{2}
$$

where $\boldsymbol{R} \in C^{N_{F F T} \times M_{F F T}}$ is 2-D periodogram of $\boldsymbol{F}$ and DFT size is $N_{F F T} \times M_{F F T}$. Usually, $N_{F F T}$ and $M_{F F T}$ are chosen as integer multiples of $N$ and $M$ to improve estimation resolution. $\boldsymbol{W}$ is a window matrix generated by

$$
\boldsymbol{W}=\boldsymbol{w}_{N} \boldsymbol{w}_{M}^{T}, \boldsymbol{w}_{N} \in R^{N \times 1}, \boldsymbol{w}_{M} \in R^{M \times 1}
$$

where $\boldsymbol{w}_{N}$ and $\boldsymbol{w}_{M}$ are one-dimensional window vectors and we uses the Hanning window. If the target's range and Doppler frequency are limited within certain ranges, only a cropped region of periodogram $\boldsymbol{R}$ is useful for target detection. Detecting and identifying targets corresponds to the detection of peaks in the periodogram. If a peak is found at indices $(\hat{n}, \hat{m})$, the target distance is $\hat{d}=\frac{c_{0} \hat{n}}{2 \Delta f N_{F F T}}$ and the relative speed is $\hat{v}=\frac{c_{0} \hat{m}}{2 f_{C} T M_{F F T}}$. Due to the subcarrier spacing $\Delta f$ and the OFDM symbol duration $T$, there are maximum unambiguous ranges and relative velocities as follows

$$
\begin{aligned}
& \left|d_{\max } \| \frac{c_{0}}{2 \Delta f}\right| \\
& \left|v_{\max }\right|\left|\frac{c_{0}}{2 f_{C} T}\right|
\end{aligned}
$$

If the subcarrier spacing and the OFDM symbol duration are designed to be small enough, the maximum unambiguous values can cover the target's distance and speed.

\section{Proposed method}

This paper focuses on detecting the number of the targets. First, we describe the conventional method and then the proposed method is explained.

3.1 Conventional method

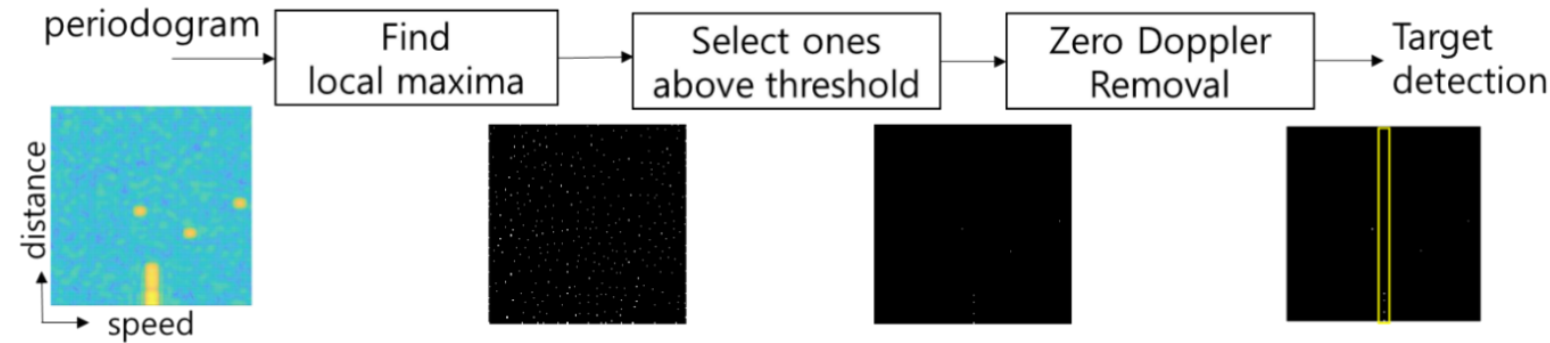

Figure 2 Block diagram of conventional target detection

As shown in Figure 2, the conventional method find all the local maxima first. Among them, those ones above a certain threshold are selected. In this procedure, only big peaks remain and small peaks are discarded. However, the big direct coupling components still remain and further process is required to remove them. One simple way is to neglect peaks around zero Doppler frequency. After removing direct coupling components, the remaining peaks are considered as the targets. Therefore, the number of targets is the number of remaining peaks. 
However, during the zero Doppler removal procedure, the targets whose relative speed is close to zero, may also be discarded and not be detected as targets. This causes detection errors. In addition, since the optimal threshold is a function of SNR and noise variance of the reflected signal, determining optimal threshold in real field applications is rather difficult.

\subsection{Proposed method}

The proposed method predicts the number of target directly from the periodogram through CNN classifier. $\mathrm{CNN}$ is a famous deep learning technique that shows excellent performance especially in image classification. As shown in Figure 2, the periodogram can be an image and the radar problem is to find the peaks in the image. Therefore, it is expected that the CNN is very suitable for our radar problem. Figure 3 shows the block diagram of the proposed CNN classifier. The input signal is a 200x200 cropped periodogram. As mentioned previously, if the target's maximum range and speed is limited, we may search only a cropped region. As search range increases, the crop size also increases.

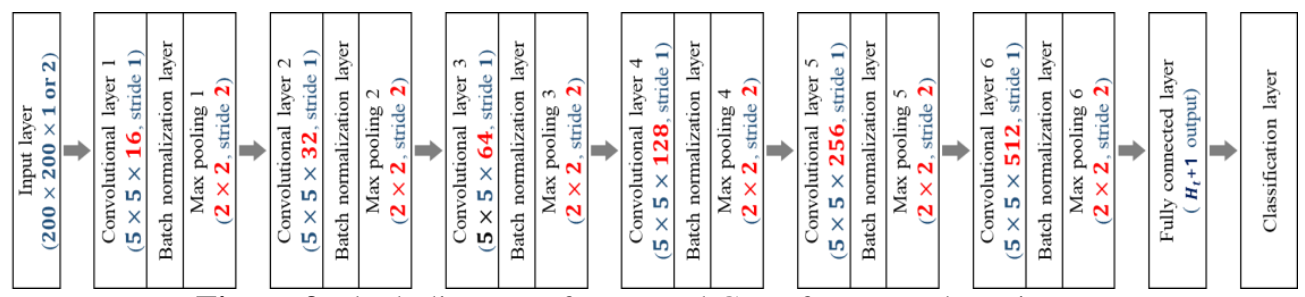

Figure 3 Block diagram of proposed CNN for target detection

The proposed CNN classifier is composed of 6 convolutional layers and 1 fully connected layer. The convolution filter size is $5 \times 5$ for every convolutional layer and the channel (or kernel) size is increased from 16 to 256. Each convolution layer also has batch normalization layer, Relu layer and max pooling layer. The last convolution layer's output is connected to a fully connected layer, and the final number of output is $H_{t}+1$. This CNN classifier can detect maximum $H_{t}+1$ targets, and the possible classes are ' 0 ' to ' $H_{t}+1$ '. ' 0 ' means no target exist. The input or output of the CNN can be changed according to the OFDM signals, 2-D DFT size, and the detection range.

If the periodogram for the direct coupling components is available, the detection performance can be further improved. For example, if the radar system is equipped in a base station and is fixed in one place, the direct coupling components can be measured in the early morning when no target exists. To utilize this extra periodogram in the radar problem, we increase the input size of the CNN in Figure 3 to $200 \times 200 \times 2$. Thus, two $200 \times 200$ periodograms become the inputs of the CNN. Beside the input size, the rest of the CNN structure is the same. Figure 4 shows an example input of this scenario. The left periodogram is from the direct coupling components and the right periodogram is normal periodogram containing both the targets and the direct coupling components.

The proposed CNN classifier does not need SNR estimation nor noise variance estimation, and also, any specific peak search algorithm is not needed. Hence, it is simpler to use in practice than the conventional technique[14-16].
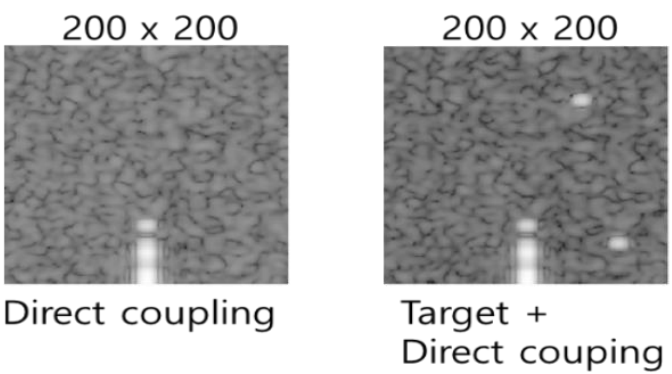

Figure 4 Periodogram examples of direct coupling only (left) and target plus direct coupling (right)

\section{Simulation result}

\subsection{Simulation environments}

Performance of the proposed $\mathrm{CNN}$ is examined through computer simulation using MATLAB. In the simulation, $f_{S}=122.88 \mathrm{MHz}, N=4096, M=64, N_{F F T}=4096$, and $M_{F F T}=256$. The length of CP is 296 and hence the symbol duration is $T=35.74 \mu \mathrm{s}$. The subcarrier spacing is $\Delta f=30 \mathrm{kHz}$. By considering $200 \mathrm{x}$ 200 cropped periodogram, the maximum identifiable distance and speed are $244 \mathrm{~m}$ and $211 \mathrm{~km} / \mathrm{h}$, respectively. To expand the detection range, we can just increase the cropped region. Among the total 4096 subcarriers, only 1,284 subcarriers are used and the signal bandwidth is $40 \mathrm{MHz}$. The carrier frequency is $f_{C}=28 \mathrm{GHz}$. 
The maximum number of target, $H_{t}$, is 2,4 , and 8 . At each iteration, the number of target is selected randomly from 0 to $H_{t}$. For modeling the direct coupling components in (3), the number of direct coupling components is $20\left(H_{d}\right)$, and the parameters of the Weibull distribution are $\alpha=1$ and $\beta=1$ in (5). Thus, 20 direct coupling components are generated with random effective distances, and in Figure 3 , you can see the direct coupling components generated in this way. Due to the characteristic of the Weibull distribution, the effective distances are concentrated near zero.

\subsection{Training the CNN classifier}

For training the CNN classifier in Figure 3, total 50,000 periodograms are generated and used. The reflected target signals have random SNR from $-20 \mathrm{~dB}$ to $+30 \mathrm{~dB}$. The mini batch size is 100 and training is performed for 20 epochs. Therefore, the total number of parameter updates is 10,000. The parameter optimization algorithm is Adam with learning rate 0.001 . We will call ' $\mathrm{T}$ ' for one input $\mathrm{CNN}$ (target), and ' $\mathrm{T}+\mathrm{D}$ ' for two input CNN (target + direct coupling). The parameters for each layer are summarized in Table 1 for ' $T$ '. The total

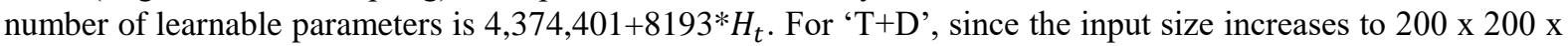
2 , the total number of parameters also increases to $4,374,801+8193 * H_{t}$.

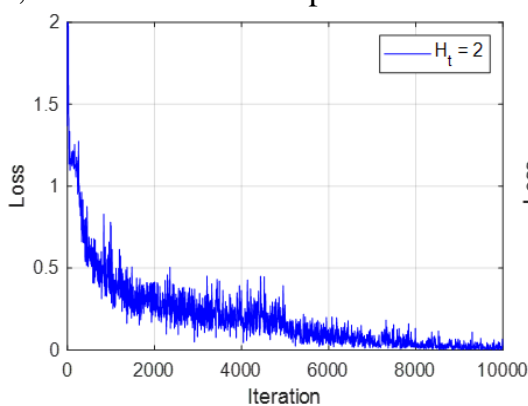

(a)

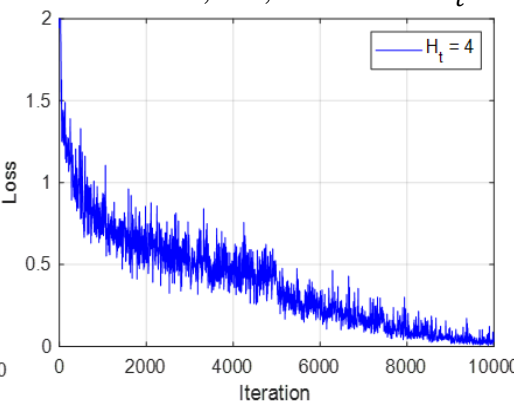

(b)

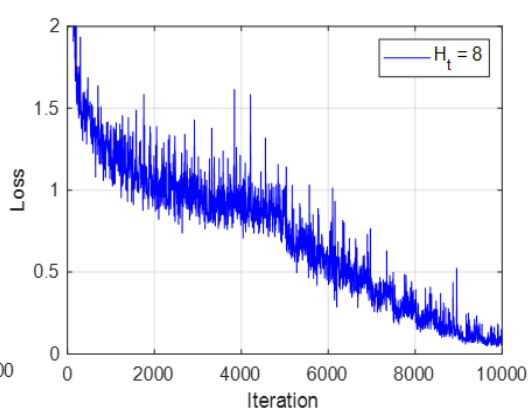

(c)

Figure 5 Learning curves for ' $T$ ' when (a) $\boldsymbol{H}_{t}=\mathbf{2}$ (b) $\boldsymbol{H}_{\boldsymbol{t}}=\mathbf{4}$ (c) $\boldsymbol{H}_{\boldsymbol{t}}=\mathbf{8}$

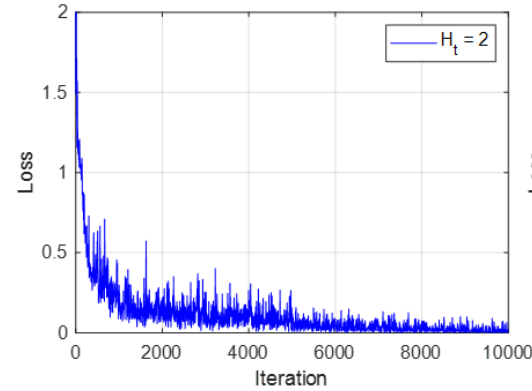

(a)

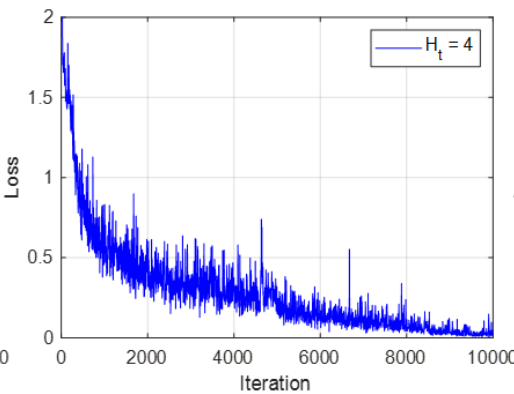

(b)

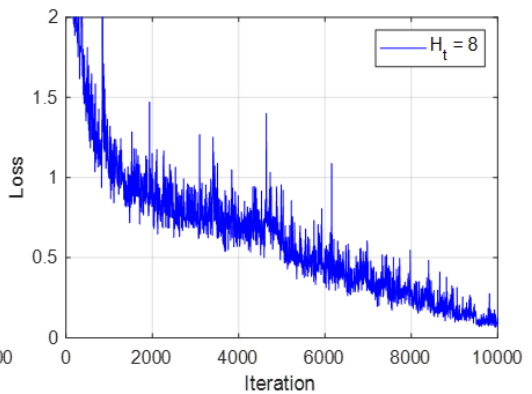

(c)

Figure 6 Learning curves for ' $\mathrm{T}+\mathrm{D}$ ' when (a) $\boldsymbol{H}_{\boldsymbol{t}}=\mathbf{2}$ (b) $\boldsymbol{H}_{\boldsymbol{t}}=\mathbf{4}$ (c) $\boldsymbol{H}_{\boldsymbol{t}}=\mathbf{8}$

Figures 5 and 6 show the learning curves for ' $\mathrm{T}$ ' and ' $\mathrm{T}+\mathrm{D}$ ', respectively. The maximum possible numbers of the targets, $H_{t}$, are 2, 4, and 8. As shown in the figures, as $H_{t}$ increases convergence time becomes longer. The reason is that as $H_{t}$ increases, so do the learnable parameters. At the end of the iteration, the losses are close to zero, which indicates that the training is completed well. Comparing ' $T$ ' and ' $T+D$ ', convergence speed of ' $\mathrm{T}+\mathrm{D}$ ' is slightly faster than ' $\mathrm{T}$ '.

\subsection{Performance comparison}

For performance evaluation of the proposed technique, new test signals are generated. For each periodogram, the number of targets is generated randomly from 0 to $H_{t}$, but the SNRs of the targets are the same. The SNR is varied from $-20 \mathrm{~dB}$ to $+10 \mathrm{~dB}$ with $3 \mathrm{~dB}$ step. At each SNR, 10,000 periodograms are generated. The Figures 7 and 8 show the accuracies of the proposed technique for ' $\mathrm{T}$ ' and ' $\mathrm{T}+\mathrm{D}$ ' respectively. As the SNR increases, the accuracy also improves. $H_{t}=2$ shows the best performance, i.e., the number of maximum targets has a big impact on accuracy. For the case ' $\mathrm{T}$ ', the accuracy is below 0.9 at best. When $H_{t}=8$, the accuracy is only 0.45 at $\mathrm{SNR}=10 \mathrm{~dB}$. In contrast, ' $\mathrm{T}+\mathrm{D}$ ' shows much better performance. When $H_{t}=2$, the accuracy at $\mathrm{SNR}=10 \mathrm{~dB}$ shows almost 1 . Even for $H_{t}=4$ and $H_{t}=8$, the performance is much better than ' $\mathrm{T}$ '. Those results indicate that if the periodogram of the direct coupling components is used, much better target detection performance can be expected. 


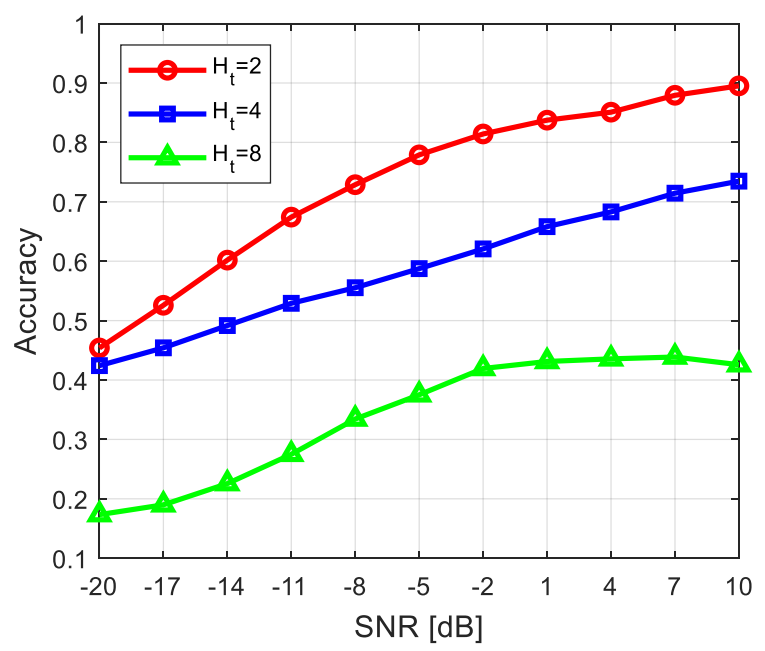

Figure 7 Accuracy performance for ' $T$ '

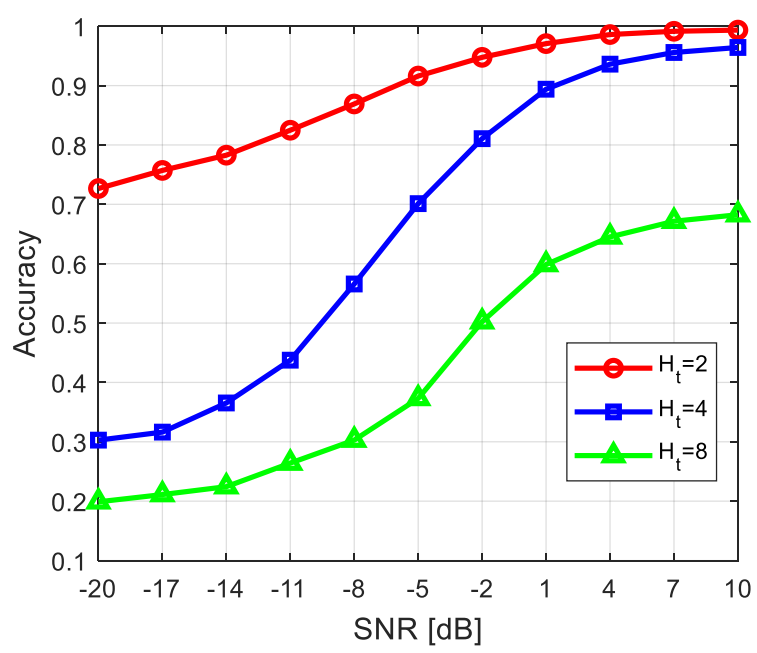

Figure 8 Accuracy performance for ' $T+D$ '

\section{Acknowledgements}

This work was supported by Institute of Information \& communications Technology Planning \& Evaluation (IITP) grant funded by the Korea government(MSIT) (No.1711081183, Development of CommunicationSensing Converged B5G Millimeter Wave System)

\section{References}

1. Strum C, Pancera E, Zwick T, Wiesbeck W. A novel approach to OFDM radar processing. Radar Conference; 2009 May 4-8; Pasadena, CA, US. IEEE; 2019. p. 1-4.

2. Strum C, Zwick T, Wiesbeck W, Braun M. Performance verification of symbol-based OFDM radar processing. Radar Conference; 2010 May 10-14; Washington, DC, US. IEEE; 2010. p. 60-63.

3. Braun M, Muller M, Fuhr M, Jondral FK. A USRP-based testbed for OFDM-based radar and communication system. Proceedings of 22nd Virginia Tech Symposium on Wireless Communications; 2012 June; 1-6.

4. Evers A, Jackson JA. Analysis of an LTE waveform for radar applications. Radar Conference; 2014 May 19-23; Cincinnati, OH, US. IEEE; 2014. p.0200-5.

5. Barneto CB, Riihonen T, Turunen M, Anttila L, Fleischer M, Stadius K, et al. Full-duplex OFDM radar with LTE and 5G NR waveforms: challenges, solutions, and measurements. IEEE Transactions on Microwave Theory and Techniques. 2019;67:4042-54.

6. Gao T, Lai Z, Mei Z, Wu Q. Hybrid SVM-CNN classification technique for moving targets in automotive FMCW radar system. 11th International Conference on Wireless Communications and Signal Processing. 2019 Oct 23-25; Xi'an, China. IEEE; 2019. p. 1-6. 
7. Heng L, Xiao L, Zeng LI, Jian LI. Radar target discrimination with F-CNN: fewer parameters and higher accuracy. 3rd International Conference on Signal and Image Processing. 2018 July 13-15; Shenzhen, China. IEEE; 2018. p.499-503.

8. Dai Y, Jin T, Song Y, Du H, Zhao D. CNN-based multiple-input multiple-output radar image enhancement method. Journal of Engineering. 2019;2019:6840-4.

9. Gao J, Deng B, Qin Y, Wang H, Li X. Enhanced radar imaging using a complex-valued convolutional neural network. IEEE Geoscience and Remote Sensing Letters. 2019;16:35-9.

10. Joung JG, Jung SH, Chung SM, Jeong ER. CNN-based Tx-Rx distance estimation for UWB system localization. Electronics Letters. 2019;55:938-40.

11. Zhao ZQ, Zheng P, Xu ST, Wu X. Object detection with deep learning: a review. IEEE Transactions on Neural Networks and Learning Systems. 2019;30:3212-32.

12. Wu X. Sahoo D. Hoi SC. Recent advances in deep learning for object detection. Neurocomputing. 2020;396:39-64

13. Wang L, Tang J, Liao Q. A study on radar target detection based on deep neural networks. IEEE Sensors Letters. 2019;3:1-4.

14. Bhoi, A. K., Mallick, P. K., Kumar, S. S., \& Sherpa, K. S. Brain tumor detection: a comparative analysis of edge detection techniques. International Journal of Applied Engineering Research, 10(44), 2015.

15. Satapathy, S. K., Mishra, S., Sundeep, R. S., Teja, U. S. R., Mallick, P. K., Shruti, M., \& Shravya, K. (2019). Deep learning based image recognition for vehicle number information. International Journal of Innovative Technology and Exploring Engineering, 8, 52-55.

16. Satapathy, S.K., Mishra, S., Mallick, P.K. et al. ADASYN and ABC-optimized RBF convergence network for classification of electroencephalograph signal. Pers Ubiquit Comput (2021). https://doi.org/10.1007/s00779-021-01533-4 